\title{
Effects of L-carnitine combined with pancreatic kininogenase on thioredoxin 2, thioredoxin reductase 1, and sperm quality in patients with oligoasthenospermia
}

\author{
Yang Wang ${ }^{1}$, Rui Zhang ${ }^{2}$, Weijun Pan $^{3}$, Zhe Xu' ${ }^{1}$, Huan Yang ${ }^{1}$, Qi Luo ${ }^{1}$, Xiping Ye ${ }^{4}$ Xianfeng Cheng \\ ${ }^{1}$ Department of Pharmacy, Ma'anshan Maternal and Child Health Hospital, Anhui, China; ${ }^{2}$ Department of Andrology, Ma'anshan Maternal \\ and Child Health Hospital, Anhui, China; ${ }^{3}$ Department of reproductive center, Ma'anshan Maternal and Child Health Hospital, Anhui, China; \\ ${ }^{4}$ Department of Reproduction laboratory, Ma'anshan Maternal and Child Health Hospital, Anhui, China; ${ }^{5}$ Department of Laboratory Medicine, \\ Institute of Dermatology, Chinese Academy of Medical Sciences and Peking Union Medical College, Nanjing, China \\ Contributions: (I) Conception and design: Y Wang; (II) Administrative support: None; (III) Provision of study materials or patients: Z Xu; (IV) \\ Collection and assembly of data: R Zhang, H Yang; (V) Data analysis and interpretation: Q Luo, X Cheng; (VI) Manuscript writing: All authors; (VII) \\ Final approval of manuscript: All authors. \\ Correspondence to: Xianfeng Cheng. Department of Laboratory Medicine, Institute of Dermatology, Chinese Academy of Medical Sciences and Peking \\ Union Medical College,12 Jiangwangmiao Street, Xuanwu District, Nanjing 210042, China. Email: cxf1979@vip.163.com.
}

Background: To study the effects of L-carnitine (LC) combined with pancreatic kininogenase on thioredoxin 2 ( $\operatorname{Tr} x 2)$, thioredoxin reductase 1 ( $\operatorname{Trx} R 1)$, and sperm quality in patients with oligoasthenospermia.

Methods: A total of 300 male infertility patients with oligoasthenospermia who were treated in the andrology clinic of our hospital from December 2019 to December 2020 were randomly divided into an LC group and combined treatment group, and 50 males with normal semen were selected as a control group. The computer-assisted semen analysis system (CASA) was used to detect the total number, vitality, and forward motility of the sperm before and after treatment, and sperm morphology was detected by the DiffQuik method of the sperm staining kit. Sperm chromatin dispersion (SCD) method was used to detect sperm DNA fragments, and Western-blot was used to detect the protein expression of Trx 2 and TrxR 1.

Results: There were no significant differences in sperm density, motility rate, forward motile sperm rate, and DNA fragmentation rate in oligoasthenospermia patients before treatment $(\mathrm{P}>0.05)$. However, after 1 month of treatment, the sperm density, motility rate, and forward motile sperm rate were all higher than before treatment $(\mathrm{P}<0.05)$, while the DNA fragmentation rate was lower than before treatment. At the same time, each index of semen in the combination group was higher than that in the LC group $(\mathrm{P}<0.05)$, and the total effective rate in the combination group was significantly higher than in the LC group $(\mathrm{P}<0.01)$. The expression of Trx 2 protein in oligoasthenospermia patients was significantly increased $(\mathrm{P}<0.05)$, while the expression of TrxR1 protein was significantly decreased $(\mathrm{P}<0.05)$. After 3 months of treatment, the expression of $\operatorname{Tr} x 2$ protein was significantly decreased $(\mathrm{P}<0.05)$, while the expression of TrxR1 protein was significantly increased $(\mathrm{P}<0.05)$.

Conclusions: The results suggest Trx 2 and TrxR 1 may be candidate protein markers for oligoasthenospermia. LC combined with pancreatic kininogenase in the treatment of male oligoasthenospermia can effectively promote sperm maturation, enhance sperm motility, and improve semen quality, which has high application value.

Keywords: L-carnitine (LC); pancreatic kininogenase; oligoasthenospermia; thioredoxin 2 (Trx 2); thioredoxin reductase $1(\operatorname{Trx} R$ 1)

Submitted Jun 17, 2021. Accepted for publication Aug 18, 2021.

doi: $10.21037 /$ tau-21-680

View this article at: https://dx.doi.org/10.21037/tau-21-680 


\section{Introduction}

Infertility seriously affects human reproductive health and family harmony, and brings a heavy burden to society. The incidence of infertility is increasing year by year, and among married people is as high as $10-15 \%$, of which male factors account for $50 \%(1,2)$. Epidemiological studies suggest oligoasthenospermia is an important cause of male infertility. According to the 5th edition of Semen Parameters, oligozoospermia is indicated when sperm density is less than $15 \times 10^{6} / \mathrm{mL}$; asthenospermia is indicated when the composition ratio of sperm with fast forward motility is less than $32 \%$; and when both sperm density and fast forward motility of sperm are lower than normal, oligoasthenospermia is indicated (3).

In recent years, related studies have found that a high concentration of reactive oxygen species (ROS) produced by inflammatory cells, germ cells, and abnormal sperm cells during growth and metabolism often causes damage to cellular DNA, lipid, and protein, and reduces sperm concentration and vitality, which is related to the occurrence of oligoasthenospermia (4). Continuously high levels of ROS can cause oxidative stress on cells and mitochondrial function disorders (5). L-carnitine (LC) is the only carrier for the transport of long-chain fatty acids into the mitochondrial inner membrane for $\beta$-oxidation, promoting oxidation reaction and providing energy for cells (6). LC also regulates testicular supporting cells, removing oxygen free radicals in seminal plasma, reducing sperm aggregation, and reducing spermatogenic cell apoptosis $(6,7)$. At present, many hospitals regard LC as the preferred drug for the treatment of male infertility syndrome (8). The human body has a high tolerance to LC, and most patients can achieve stable absorption of the drug by direct oral administration. Pancreatic kininogenase is a proteolytic enzyme with its highest content in the pancreas. In human genitalia, pancreatic kininogenase can improve the quality of life of sperm, balance the content of components in the epididymis, and eliminate bacteria, all of which facilitate normal erectile function and regulate male sexual function (9).

This study aimed to observe and analyze the effects of LC, pancreatic kininogenase, and their combined application on thioredoxin 2 ( $\operatorname{Trx} 2)$, thioredoxin reductase $1(\operatorname{Tr} x \mathrm{R} 1)$, and sperm quality in oligoasthenospermia patients through a clinical randomized controlled study. Evaluating the efficacy and safety of the combined application of LC and pancreatic kininogenase in the treatment of oligoasthenospermia patients will establish a clinical reference for drug therapy in male patients with oligoasthenospermia. We present the following article in accordance with the STROBE reporting checklist (available at https://dx.doi.org/10.21037/tau-21-680).

\section{Methods}

\section{Research subjects}

A total of 300 male infertility patients with oligoasthenospermia who were admitted to the andrology clinic of our hospital from December 2019 to December 2020 were included in this study. Inclusion criteria: (I) the couple lived together for more than 1 year, had a normal sex life without contraception, and female fertility test results were normal; (II) patients stopped all spermatogenic drug treatments for $\geq 12$ weeks before the start of the study; and (III) following the fifth edition of the WHO standard, the male was diagnosed as oligozoospermia (sperm concentration $<15 \times 10^{6} / \mathrm{mL}$ ) or asthenospermia (forward motility $<32 \%$ ) when examined more than twice. Exclusion criteria: (I) patients with significant systemic disease, endocrine disease, reproductive tract infection, and varicocele, and (II) patients with a history of cryptorchidism, orchitis, mumps in late adolescence, anti-sperm antibodies, or other known etiologies of male infertility. All procedures performed in this study involving human participants were in accordance with the Declaration of Helsinki (as revised in 2013). The study was approved by Ma'anshan Maternal and Child Health Hospital (No. 2019084). Each participant was informed in detail about the content of the study and their rights, and participated voluntarily. All enrolled patients signed an informed consent form.

Patients were randomly divided into an LC group and combined treatment group, each composed of 150 patients. Patients in the LC group ranged from 22-34 years, with an average age of $27.85 \pm 5.18$ years, while those in the combined treatment group ranged from 20-35 years old, with an average age of $28.49 \pm 5.46$ years. A further 50 males with normal semen were selected as a control group, in which men with reproductive tract infections, sexually transmitted diseases, blocked vas deferens, tumors, trauma surgery, and diabetes were excluded.

\section{Treatment methods}

The LC group were given LC oral solution (10 mL: $1 \mathrm{~g})$, $10 \mathrm{~mL} /$ time, three times per day, taken with meals (Shenyang First Pharmaceutical Co., Ltd., Northeast 
Pharmaceutical Group). In the combined treatment group, a pancreatic kininogenase enteric-coated tablet was added, and was taken as $120 \mathrm{U}$, three times per day.

\section{Test indexes}

Semen samples were collected 3-7 days after the cessation of sexual activity before treatment, and 1 and 3 months after treatment, and all samples were stored in an incubator at $37^{\circ} \mathrm{C}$. After liquefaction, the sperm count, motility, and forward mobility were detected by the computer-assisted semen analysis system (CASA) in accordance with the testing standards of the Human Semen Examination and Processing Laboratory Manual formulated by the WHO. The sperm motility rate and forward motility rate were calculated as follows: sperm motility rate $=$ motile sperm (forward motile sperm + non-forward motile sperm)/total sperm count $\times 100 \%$; forward motile sperm rate $=$ forward motile sperm count/total sperm count $\times 100 \%$. Sperm morphology detection was performed using the Diff-Quik method described in the instructions of the sperm staining kit (Shenzhen Boride Biotechnology Co., Ltd.), and sperm DNA fragments were detected by the sperm chromatin dispersion (SCD) method. Kits were obtained from Anke Biotech Co., Ltd. The sperm DNA fragmentation rate = (halo-free sperm number/total sperm number) $\times 100 \%$.

\section{Clinical efficacy}

According to the relevant criteria [8], cure was defined as pregnancy occurring normally during treatment or within 6 months after the treatment, and semen parameters were completely restored to normal; markedly effective was defined as sperm density $\geq 15 \times 10^{6} / \mathrm{mL}$, forward motile sperm $\geq 32 \%$, total sperm motility $\geq 40 \%$, but pregnancy did not occur; effective was defined when at least one of sperm density, motility, or motility rates improved $>30 \%$ compared to before treatment; and non-effective when there were no changes in semen examination parameters or even deterioration. The total effective rate $=($ cure + markedly effective + effective) cases/total cases $\times 100 \%$.

\section{Western-blot}

Sperm in both groups was extracted with RIPA lysate, and the protein concentration was determined. A $5 \times$ loading buffer was then added, and the mixture boiled for $10 \mathrm{~min}$ to denature the sperm. According to the molecular weight of the target protein, the separation glue and the concentrate glue were configured, and the sample was loaded to start electrophoresis at $80 \mathrm{~V}$. After 30 minutes, the sample was concentrated into the separation gel, and the voltage was changed to $120 \mathrm{~V}$ for 90 minutes. The PVDF membrane activated by methanol was used for transfer for 2 hours, then sealed with $5 \%$ skim milk for 2 hours, after which it was cut according to the size of the target protein band and incubated in rabbit anti-human $\operatorname{Trx} 2$ (1:300) and $\operatorname{Trx} R 1$ (1:300) antibodies at $4{ }^{\circ} \mathrm{C}$ overnight. The second day, the membrane was removed, washed with TBST for three times, and the secondary antibody $(1: 6,000)$ was used for incubation at room temperature for 2 hours. This was again washed with TBST three times. Solution A and solution B in the ECL detection kit were mixed in a 1:1 ratio and dropped into the PVDF membrane, and observed by gel imager.

\section{Statistical method}

SPSS 20.0 statistical software was used and, the measurement data were represented as $\bar{x} \pm \mathrm{s}$ and tested by $t$ test. Chi-square test was used for counting data (\%), and if the theoretical frequency $<1$, Fisher's exact test was used, and the rank sum test was used for rank counting data. $\mathrm{P}<0.05$ indicated a statistically significant difference.

\section{Results}

\section{Changes of parameters and indexes in routine semen examination}

All patients completed the study. There were no significant differences in sperm density, motility rate, forward motile sperm rate, and DNA fragmentation rate in oligoasthenospermia patients before treatment $(\mathrm{P}>0.05)$. However, after 1 month of treatment, the sperm density, motility rate, and forward motile sperm rate were all higher than those before treatment $(\mathrm{P}<0.05)$, while the DNA fragmentation rate was lower than that before treatment. At the same time, the parameters of semen in the combination group were higher than those in the LC group $(\mathrm{P}<0.05)$. After 3 months of treatment, semen parameters improved significantly, as shown in Table 1 and Figure 1. The morphology of the sperm is shown in Figure 2.

\section{Clinical efficacy}

The clinical efficacy of patients after 1 month and 3 months 

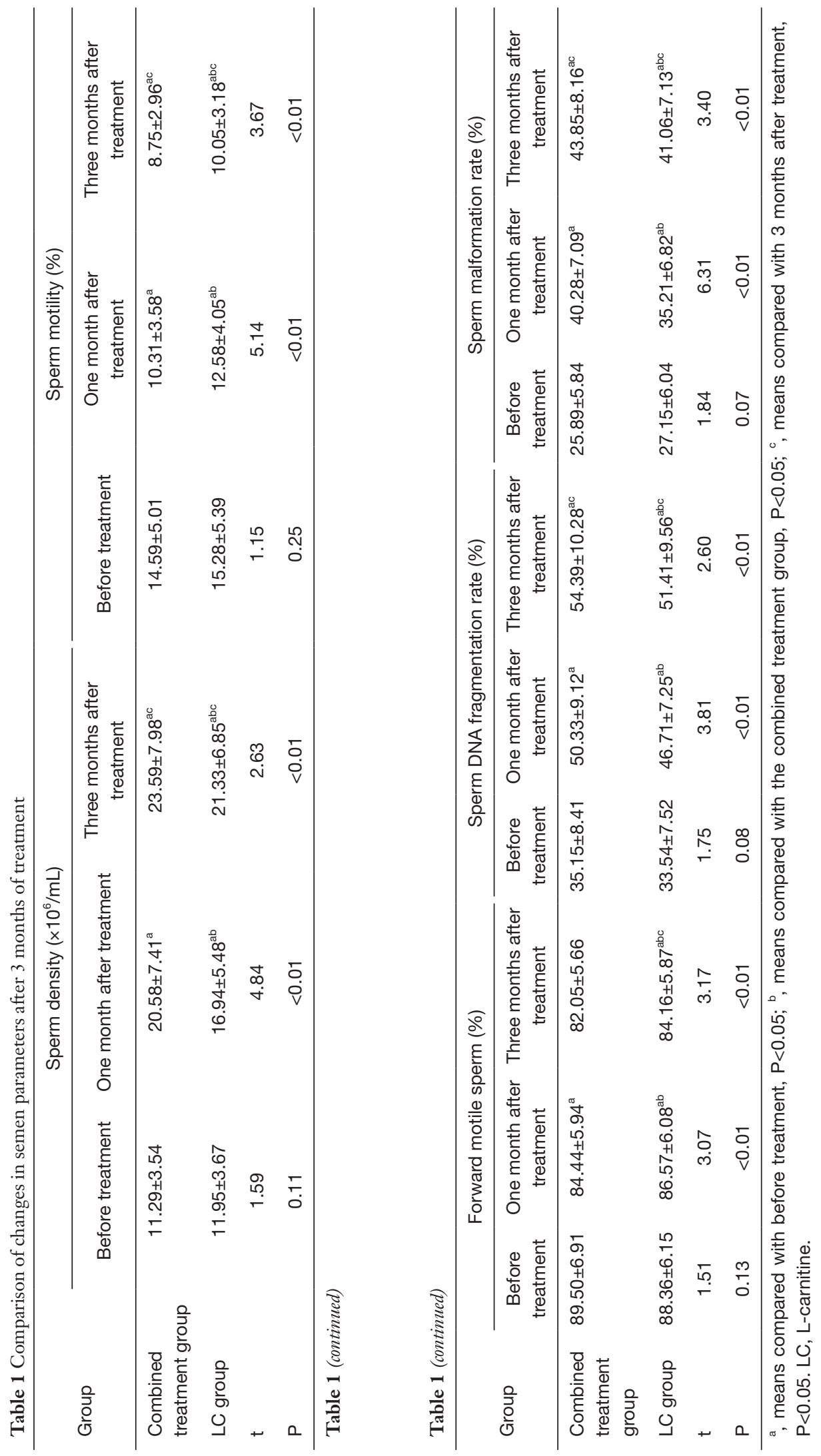


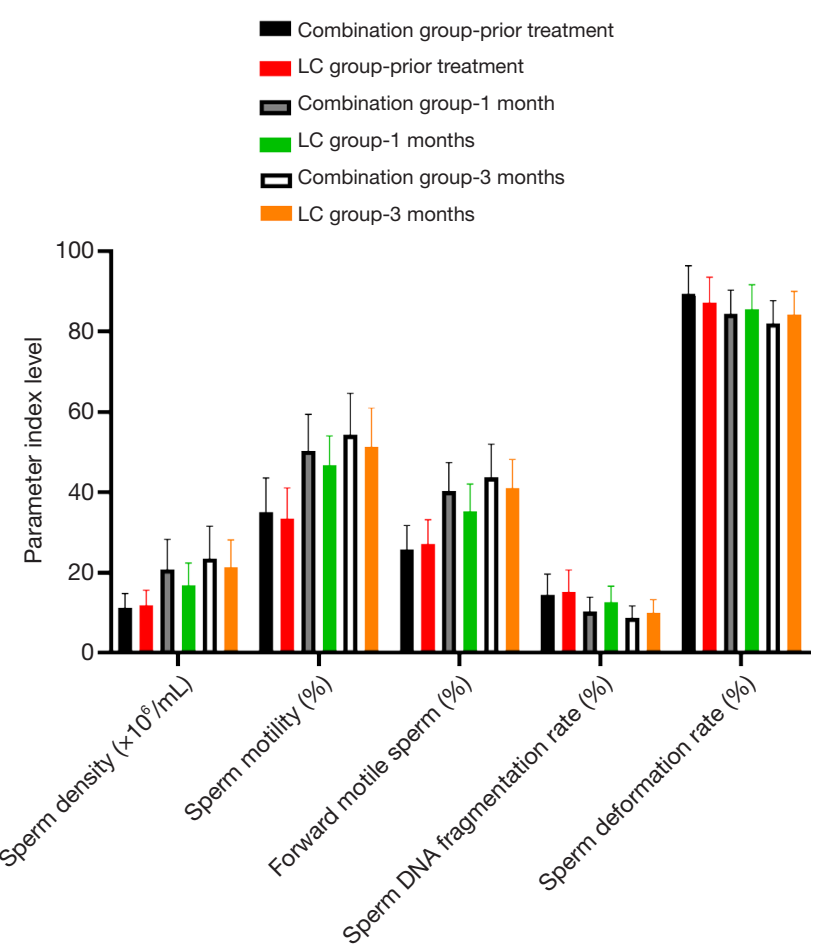

Figure 1 Comparison of semen parameters before and after treatment. LC, L-carnitine.

of treatment was recorded, respectively. The results showed that the markedly effective rate of patients in the combined treatment group was significantly higher than that in the LC group $(\mathrm{P}<0.05)$, and the total effective rate of patients in the combined treatment group was significantly higher than that in the LC group $(\mathrm{P}<0.01)$, as shown in Table 2.

\section{Expression of Trx 2 and TrxR1 in sperm}

Western-blot results showed that before treatment, compared with men with normal semen, the expression of $\operatorname{Tr} x 2$ protein in oligoasthenospermia patients was significantly increased $(\mathrm{P}<0.05)$, while the expression of TrxR1 protein was significantly decreased $(\mathrm{P}<0.05)$. After 1 month of treatment, the fluorescence intensity of $\operatorname{Tr} x 2$ protein was decreased and that of TrxR1 protein was increased, but there was no significant difference between the LC group and the combined treatment group $(\mathrm{P}>0.05)$. After 3 months of treatment, the fluorescence intensity of Trx2 protein was significantly down-regulated compared with before treatment $(\mathrm{P}<0.05)$, while that of TrxR1 protein was significantly up-regulated $(\mathrm{P}<0.05)$, as shown in Table 3 and Figures 3 and 4.

\section{Discussion}

Male oligoasthenospermia is one of the main causes of male infertility. In recent years, due to environmental deterioration and lifestyle changes, the incidence of this disease has increased, which has a great impact on male physical and mental health and family harmony (10). How to effectively treat oligoasthenospermia has become a hot spot in clinical research. At present, drug therapy is the main treatment, and as there are many clinically related medications available, the choice of drug regimen is important. According to the treatment method, it can be divided into Chinese medicine treatment and western medicine treatment. Traditional Chinese medicine relies on its overall view and syndrome differentiation system, and treats people according to syndrome and cause. It has certain advantages in treating oligoasthenospermia and has significant curative effect, but the effect is not ideal for severe oligoasthenospermia caused by genetic factors. Western medicine treatment of oligoasthenospermia mainly for the etiology of treatment, clinical use is more widely $\mathrm{L}$-carnitine, vitamin $\mathrm{E}$, and anti-estrogen drugs (Androgens, gonadotropins, anti-estrogen drugs). The clinical efficacy of these drugs alone is often not significant. In the case of multi-drug combination therapy, the clinical use of hormone drugs is limited due to the inexact efficacy and many adverse reactions. At present, there are many studies focusing on the treatment of integrated Chinese and western medicine. This study focused on western medicine.

LC in the human body mainly derives from dietary intake and biosynthesis in the brain, liver, and kidney. LC is mainly distributed in blood and tissues, among which the epididymis has the highest concentration, although it is not synthesized here (11). Studies have shown that when sperm move from the head to the tail of the epididymis, LC can reduce lipid content on the sperm membrane, and increase the ratio of saturated fatty acids, unsaturated fatty acids, cholesterol, and congealed fat, causing changes in membrane composition and structure and maintaining fluidity of the sperm membrane (7). Other reports have shown that LC has an antioxidant effect, can inhibit the production of reactive oxygen species, avoid sperm oxidative damage, and effectively maintain the normal physiological function of sperm $(12,13)$. LC is one of the preferred drugs for the treatment of male infertility, and has a certain curative effect on oligozoospermia, asthenospermia, and teratozoospermia.

Pancreatic kallikrein, also known as pancreatic kallikrein 


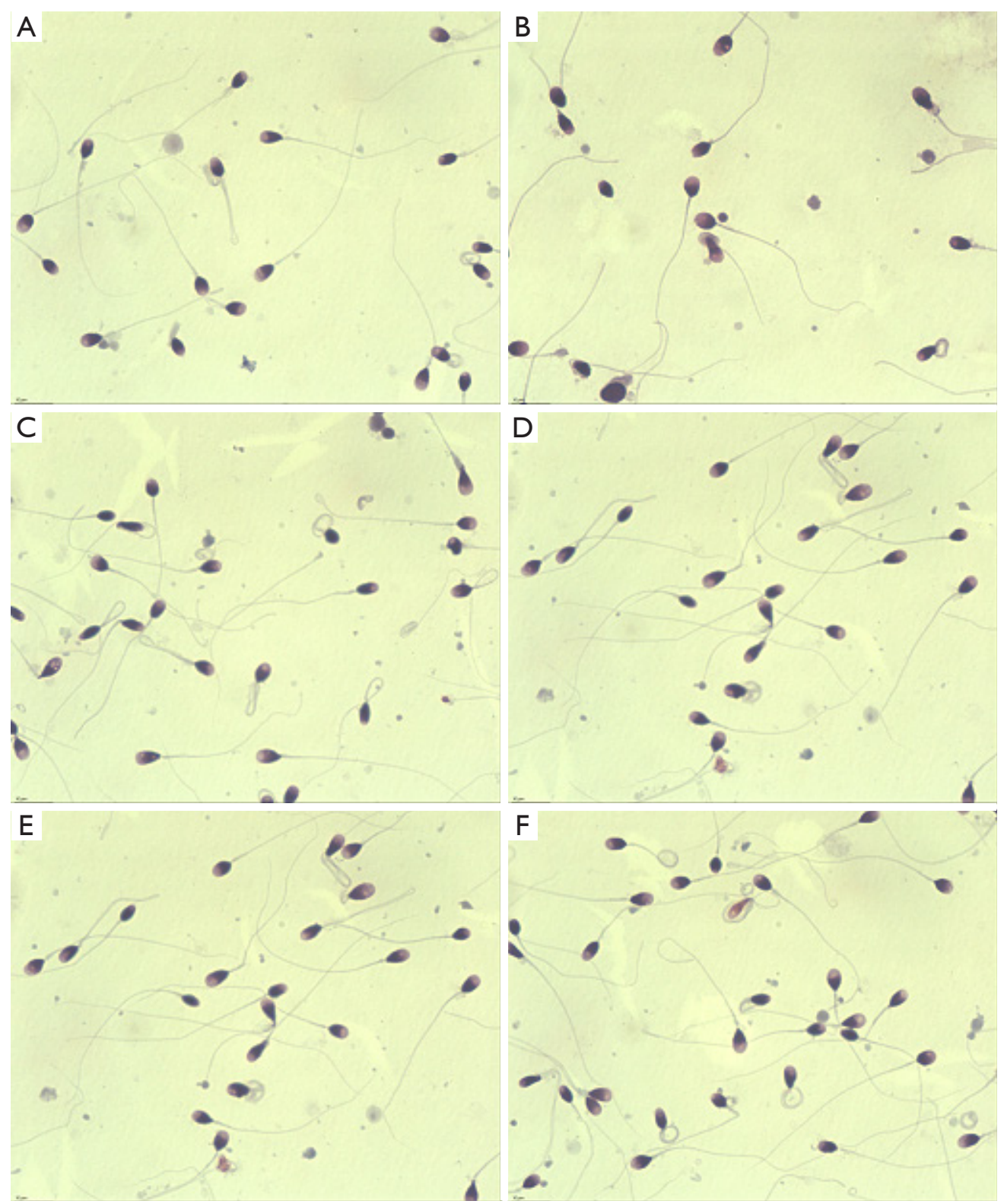

Figure 2 Sperm morphology analysis report (Diff-quik staining method, 200x). (A) Normal sperm morphology; (B) sperm morphology of patients with oligoasthenospermia; (C) sperm morphology of patients with oligoasthenospermia after one month of LC treatment; (D) sperm morcphology of patients with oligoasthenospermia after one month LC combined with pancreatic kininogenase; (E) sperm morphology of patients with oligoasthenospermia after three months of LC treatment; (F) sperm morphology of patients with oligoasthenospermia after three months LC combined with pancreatic kininogenase. LC, L-carnitine.

and kallikrein, is an important component of the kinin system. As a proteolytic enzyme, it is commonly found in the pancreas, submandibular glands, and saliva, among which the content is highest in the pancreas. The use of pancreatic kininogenase can increase protein content in the epididymis, clear blood vessels, and improve the quality of sperm (14), and studies have also confirmed that it regulates genital function. After pancreatic peptidogenase enters the genitals, its pharmaceutical effect appears immediately and penetrates every part of the genitals, restoring their vitality, improving sexual function and having an optimizing effect on the penis (15). Pancreatic peptidase has made an 
Table 2 Comparison of clinical efficacy (n, \%)

\begin{tabular}{|c|c|c|c|c|c|c|c|c|c|c|}
\hline Group & \multicolumn{2}{|c|}{ Cure } & \multicolumn{2}{|c|}{ Markedly effective } & \multicolumn{2}{|c|}{ Effective } & \multicolumn{2}{|c|}{ Non-effective } & \multicolumn{2}{|c|}{ Total effective rate (\%) } \\
\hline $\begin{array}{l}\text { Combined } \\
\text { treatment } \\
\text { group }\end{array}$ & 19 & 25 & 70 & 76 & 35 & 40 & 26 & 9 & 124 & 141 \\
\hline$P$ & 0.12 & 0.24 & 0.04 & 0.03 & 0.32 & 0.79 & $<0.01$ & $<0.01$ & - & - \\
\hline
\end{tabular}

LC, L-carnitine.

Table 3 Expression of Trx2 and TrxR1 in sperm was detected by Western-blot

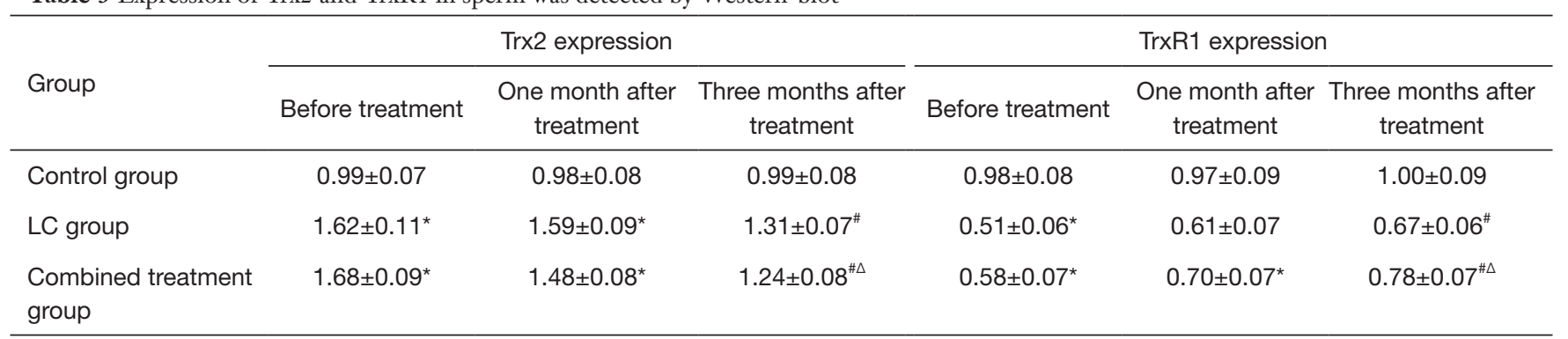

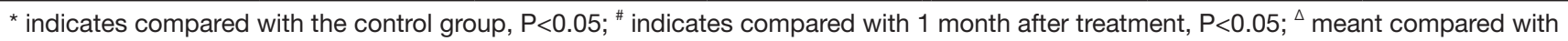
the LC group, $\mathrm{P}<0.05$. LC, L-carnitine.

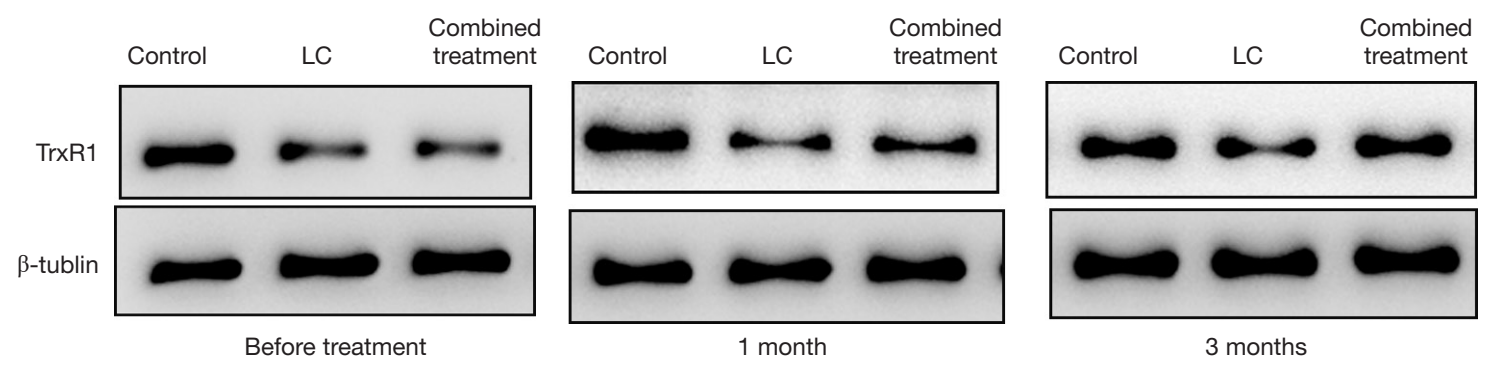

Figure 3 Expression of TrxR 1 in sperm of subjects at different times was detected by Western-blotting. LC, L-carnitine; TrxR 1, thioredoxin reductase 1 .

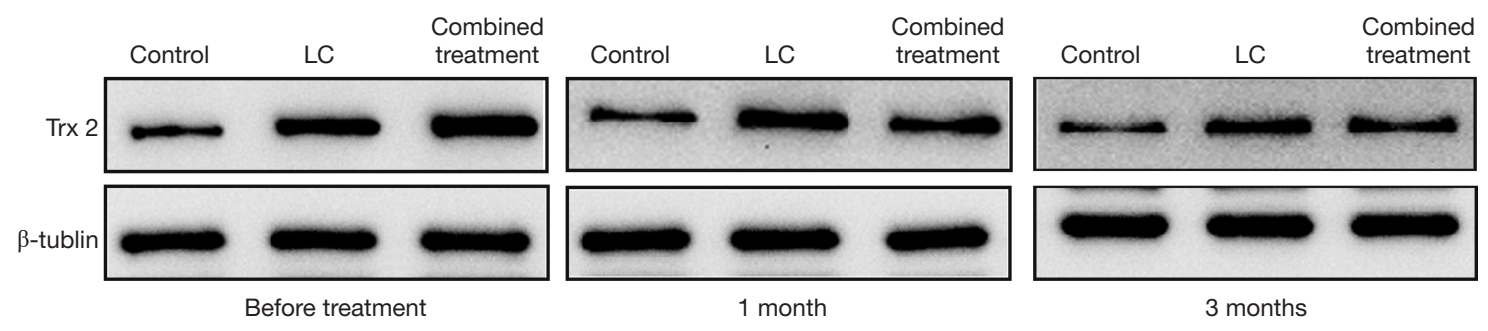

Figure 4 Expression of Trx 2 in sperm at different times was detected by Western-blotting. LC, L-carnitine; Trx 2, thioredoxin 2. 
important contribution to the treatment of male diseases $(14,16)$. It is mainly used for the treatment of varicocele (17) either alone or in combination with other drugs, and pancreatic propeptidates can be used in the treatment of male sexual dysfunction caused by diabetes. In this study, LC and pancreatic peptidogenase were used to treat male patients with oligoasthenospermia. The results showed that the total effective rate of patients in the combined treatment group was significantly higher than that of the LC group. Sperm density, sperm motility, forward motility, DNA fragmentation degree, and the sperm malformation rate after combined treatment were all better than those in the LC group, suggesting that LC and pancreatic peptidogenase have a positive synergistic effect, which can improve semen quality and enhance the therapeutic effect through different mechanisms.

The $\operatorname{Tr} x / \operatorname{Trx} R$ system exists in human sperm and plays an important role in the defense of sperm oxidative stress. Trx /TrxR reduces antioxidant proteins through $\mathrm{NADPH}$, and the latter can convert superoxide and hydrogen peroxide into $\mathrm{H}_{2} \mathrm{O}$, thereby eliminating ROS and avoiding sperm damage $(18,19)$. Many studies have confirmed that when there is oxidative stress, Trx in serum or plasma is significantly increased, which is a sign of oxidative stress (20). This study found that Trx 2 in patients with oligoasthenospermia was significantly up-regulated compared with men with normal semen. This suggests that in the sperm of patients with oligoasthenospermia, as an important substance of anti-oxidative damage, the increased compensatory expression of Trx 2 can reduce the degree of oxidative stress to a certain extent. After treatment, the expression of $\operatorname{Tr} x 2$ was down-regulated, and the down-regulation effect was more obvious in the combined treatment group than in the LC group. TrxR 1 is considered an important enzyme that controls cellular REDOX status, antioxidant defense, and cellular REDOX regulation (21). Studies have shown that in asthenospermia, the content of TrxR is decreased, the content of sperm ROS is increased, sperm apoptosis is increased, and the number of immature sperm is increased (22). In this study, TrxR 1 was significantly down-regulated in oligoasthenospermia patients compared with men with normal semen, and the mechanism may be related to the increased oxidative stress level and apoptosis level of sperm caused by its low expression. After treatment, the expression of TrxR 1 was upregulated, and the upregulated effect was more obvious in the combined treatment group than in the LC group.

In conclusion, Trx 2 and Trxr 1 are abnormally expressed in oligoasthenospermia patients, which suggests they play an important role in its occurrence and development. and may be potential protein markers for the disease. However, the specific mechanism remains unknown, and requires further study. LC combined with pancreatic peptidogenase in the treatment of male oligoasthenospermia can effectively promote sperm maturation, enhance sperm motility, and improve semen quality, which has high application value.

\section{Acknowledgments}

Funding: None.

\section{Footnote}

Reporting Checklist: The authors have completed the STROBE reporting checklist. Available at https://dx.doi. org/10.21037/tau-21-680

Data Sharing Statement: Available at https://dx.doi. org/10.21037/tau-21-680

Conflicts of Interest: All authors have completed the ICMJE uniform disclosure form (available at https://dx.doi. org/10.21037/tau-21-680). The authors have no conflicts of interest to declare.

Ethical Statement: The authors are accountable for all aspects of the work in ensuring that questions related to the accuracy or integrity of any part of the work are appropriately investigated and resolved. All procedures performed in this study involving human participants were in accordance with the Declaration of Helsinki (as revised in 2013). The study was approved by Ma'anshan Maternal and Child Health Hospital (No. 2019084). Each participant was informed in detail about the content of the study and their rights, and participated voluntarily. All enrolled patients signed an informed consent form.

Open Access Statement: This is an Open Access article distributed in accordance with the Creative Commons Attribution-NonCommercial-NoDerivs 4.0 International License (CC BY-NC-ND 4.0), which permits the noncommercial replication and distribution of the article with the strict proviso that no changes or edits are made and the original work is properly cited (including links to both the formal publication through the relevant DOI and the license). See: https://creativecommons.org/licenses/by-nc-nd/4.0/. 


\section{References}

1. Starc A, Trampuš M, Pavan Jukić D, et al. INFERTILITY AND SEXUAL DYSFUNCTIONS: A SYSTEMATIC LITERATURE REVIEW. Acta Clin Croat 2019;58:508-15.

2. Choy JT, Eisenberg ML. Comprehensive men's health and male infertility. Transl Androl Urol 2020;9:S239-43.

3. Vickram AS, Kamini AR, Archana K, et al. Effects of various semen extenders on semen parameters for the purpose of human male fertility preservation. Cryo Letters 2015;36:182-6.

4. Zhu Z, Kawai T, Umehara T, et al. Negative effects of ROS generated during linear sperm motility on gene expression and ATP generation in boar sperm mitochondria. Free Radic Biol Med 2019;141:159-71.

5. Dorostghoal M, Kazeminejad SR, Shahbazian N, et al. Oxidative stress status and sperm DNA fragmentation in fertile and infertile men. Andrologia 2017. doi: 10.1111/ and.12762.

6. Mongioi L, Calogero AE, Vicari E, et al. The role of carnitine in male infertility. Andrology 2016;4:800-7.

7. Mardanshahi T, Rezaei N, Zare Z, et al. Effects of L-Carnitine on the sperm parameters disorders, apoptosis of spermatogenic cells and testis histopathology in diabetic Rats. Int J Reprod Biomed 2018;17:325-36.

8. Gamidov SI, Ovchinnikov RI, Popova AY. Double-blind, randomized placebo-controlled study of efficiency and safety of complex acetyl-L-carnitine, L-carnitine fumarate and alpha-lipoic acid (Spermactin Forte) for treatment of male infertility. Urologiia2019;(4):62-8.

9. Buhling K, Schumacher A, Eulenburg CZ, et al. Influence of oral vitamin and mineral supplementation on male infertility: a meta-analysis and systematic review. Reprod Biomed Online 2019;39:269-79.

10. Gao K, Wang ZQ, Liu XC, et al. Genetic genes associated with oligospermia, asthenospermia and teratospermia: Advances in studies. Zhonghua Nan Ke Xue 2017;23:367-71.

11. Tsampoukas G, Khan MF, Katsouri A, et al. L-carnitine as primary or adjuvant treatment in infertile patients with varicocele. A systematic review. Arch Ital Urol Androl 2020. doi: 10.4081/aiua.2020.3.263.

12. Cabral REL, Mendes TB, Vendramini V, et al. Carnitine partially improves oxidative stress, acrosome integrity, and reproductive competence in doxorubicin-treated rats. Andrology 2018;6:236-46.

13. Shafiei G, Almasi M, Nikzad H, et al. 1-carnitine reduces the adverse effects of ROS and up-regulates the expression of implantation related genes in in vitro developed mouse embryos. Theriogenology 2020;145:59-66.

14. Chen GT, Yang BB, Chen JH, et al. Pancreatic kininogenase improves erectile function in streptozotocininduced type 2 diabetic rats with erectile dysfunction. Asian J Androl 2018;20:448-53.

15. Jin JZ, Li HY, Jin J, et al. Exogenous pancreatic kininogenase protects against renal fibrosis in rat model of unilateral ureteral obstruction. Acta Pharmacol Sin 2020;41:1597-608.

16. Zhu D, Zhang L, Cheng L, et al. Pancreatic Kininogenase Ameliorates Renal Fibrosis in Streptozotocin InducedDiabetic Nephropathy Rat. Kidney Blood Press Res 2016;41:9-17.

17. Panach-Navarrete J, Morales-Giraldo A, Ferrandis-Cortés $\mathrm{C}$, et al. Is there a relationship between varicocele and testosterone levels? Aging Male 2020;23:592-8.

18. Mitozo PA, de Souza LF, Loch-Neckel G, et al. A study of the relative importance of the peroxiredoxin-, catalase-, and glutathione-dependent systems in neural peroxide metabolism. Free Radic Biol Med 2011;51:69-77.

19. Balsera M, Buchanan BB. Evolution of the thioredoxin system as a step enabling adaptation to oxidative stress. Free Radic Biol Med 2019;140:28-35.

20. Bhatt NM, Aon MA, Tocchetti CG, et al. Restoring redox balance enhances contractility in heart trabeculae from type 2 diabetic rats exposed to high glucose. Am J Physiol Heart Circ Physiol 2015;308:H291-302.

21. Engelman R, Ziv T, Arnér ESJ, et al. Inhibitory nitrosylation of mammalian thioredoxin reductase 1: Molecular characterization and evidence for its functional role in cellular nitroso-redox imbalance. Free Radic Biol Med 2016;97:375-85.

22. Moradi MN, Karimi J, Khodadadi I, et al. Evaluation of the $\mathrm{p} 53$ and Thioredoxin reductase in sperm from asthenozoospermic males in comparison to normozoospermic males. Free Radic Biol Med 2018;116:123-8.

(English Language Editor: B. Draper)

Cite this article as: Wang $\mathrm{Y}$, Zhang $\mathrm{R}$, Pan $\mathrm{W}, \mathrm{Xu} \mathrm{Z}$, Yang $\mathrm{H}$, Luo Q, Ye X, Cheng X. Effects of L-carnitine combined with pancreatic kininogenase on thioredoxin 2, thioredoxin reductase 1 , and sperm quality in patients with oligoasthenospermia. Transl Androl Urol 2021;10(8):3515-3523. doi: 10.21037/tau-21-680 